\title{
An elementary proof of the completeness of the Eukasiewicz axioms
}

\author{
Michal Botur \\ Department of Algebra and Geometry \\ Faculty of Science, Palacký University \\ tř. 17.listopadu 1192/12, 77146 Olomouc, Czech Republic \\ michal botur@upol.cz
}

The main aim of this talk is twofold. Firstly, to present an elementary method based on Farkas' lemma for rationals how to embed any finite partial subalgebra of a linearly ordered MV-algebra into Q $[0 ; 1]$ and then to establish a new elementary proof of the completeness of the Lukasiewicz axioms for which the MV-algebras community has been looking for a long time. Secondly, to present a direct proof of Di Nola's representation Theorem for MV-algebras and to extend his results to the restriction of the standard MV-algebra on rational numbers.

\section{Introduction}

The representation theory of MV-algebras is based on Chang's representation Theorem 4, McNaughton's Theorem and Di Nola's representation Theorem [5. Chang's representation Theorem yields a subdirect representation of all MV-algebras via linearly ordered MV-algebras. McNaughton's Theorem characterizes free MV-algebras as algebras of continuous, piece-wise linear functions with integer coefficients on $[0,1]$. Finally, Di Nola's representation Theorem describes MV-algebras as sub-algebras of algebras of functions with values into a non-standard ultrapower of the MV-algebra $[0,1]$.

The main motivation for our paper comes from the fact that although the proofs of both Chang's representation Theorem [4] and McNaughton's Theorem are of algebraic nature the proof of Di Nola's representation Theorem is based on model-theoretical considerations. We give a simple, purely algebraic, proof of it and its variants based on the Farkas' Lemma for rationals [6] and General finite embedding theorem [3].

\subsection{Generalized finite embedding theorem}

By an ultrafilter on a set $I$ we mean an ultrafilter of the Boolean algebra $\mathcal{P}(I)$ of the subsets of $I$.

Let $\left\{\mathbf{A}_{i} ; i \in I\right\}$ be a system of algebras of the same type $\mathrm{F}$ for $i \in I$. We denote for any $x, y \in \prod_{i \in I} A_{i}$ the set

$$
\llbracket x=y \rrbracket=\{j \in I ; x(j)=y(j)\} .
$$

If $F$ is a filter of $\mathcal{P}(I)$ then the relation $\theta_{F}$ defined by

$$
\theta_{F}=\left\{\langle x, y\rangle \in\left(\prod_{i \in I} A_{i}\right)^{2} ; \llbracket x=y \rrbracket \in F\right\}
$$

is a congruence on $\prod_{i \in I} \mathbf{A}_{i}$. For an ultrafilter $U$ of $\mathcal{P}(I)$, an algebra

$$
\left(\prod_{i \in I} \mathbf{A}_{i}\right) / U:=\left(\prod_{i \in I} \mathbf{A}_{i}\right) / \theta_{U}
$$

N. Galatos, A. Kurz, C. Tsinakis (eds.), TACL 2013 (EPiC Series, vol. 25), pp. 35-38 
is said to be an ultraproduct of algebras $\left\{\mathbf{A}_{i} ; i \in I\right\}$. Any ultraproduct of an algebra $\mathbf{A}$ is called an ultrapower of $\mathbf{A}$. The class of all ultraproducts (products, isomorphic images) of algebras from some class of algebras $\mathcal{K}$ is denoted by $\mathrm{P}_{\mathrm{U}}(\mathcal{K})(\mathrm{P}(\mathcal{K}), \mathrm{I}(\mathcal{K}))$. The class of all finite algebras from some class of algebras $\mathcal{K}$ is denoted by $\mathcal{K}_{\text {Fin }}$.

Definition 1. Let $\mathbf{A}=(A, \mathrm{~F})$ be a partial algebra and $X \subseteq A$. Denote the partial algebra $\left.\mathbf{A}\right|_{X}=(X, \mathrm{~F})$, where for any $f \in F_{n}$ and all $x_{1}, \ldots, x_{n} \in X, f^{\left.\mathbf{A}\right|_{X}}\left(x_{1}, \ldots, x_{n}\right)$ is defined if and only if $f^{\mathbf{A}}\left(x_{1}, \ldots, x_{n}\right) \in X$ holds. Moreover, then we put

$$
f^{\left.\mathbf{A}\right|_{X}}\left(x_{1}, \ldots, x_{n}\right):=f^{\mathbf{A}}\left(x_{1}, \ldots, x_{n}\right) .
$$

Definition 2. An algebra $\mathbf{A}=(A, \mathrm{~F})$ satisfies the general finite embedding (finite embedding property) property for the class $\mathcal{K}$ of algebras of the same type if for any finite subset $X \subseteq A$ there are an (finite) algebra $\mathbf{B} \in \mathcal{K}_{\mathrm{E}}$ and an embedding $\rho:\left.\mathbf{A}\right|_{X} \hookrightarrow \mathbf{B}$, i.e. an injective mapping $\rho: X \rightarrow B$ satisfying the property $\rho\left(f^{\left.\mathbf{A}\right|_{X}}\left(x_{1}, \ldots, x_{n}\right)\right)=f^{\mathbf{B}}\left(\rho\left(x_{1}\right), \ldots, \rho\left(x_{n}\right)\right)$ if $x_{1}, \ldots, x_{n} \in X, f \in \mathrm{F}_{n}$ and $f^{\left.\mathbf{A}\right|_{X}}\left(x_{1}, \ldots, x_{n}\right)$ is defined.

Finite embedding property is usually denoted by (FEP). Note also that a quasivariety $\mathcal{K}$ has the FEP if and only if $\mathcal{K}=\operatorname{ISPP}_{\mathrm{U}}\left(\mathcal{K}_{\text {Fin }}\right)$ (see [2, Theorem 1.1] or [1]).

Theorem 1. 3, Theorem 6] Let $\mathbf{A}=(A, \mathrm{~F})$ be a algebra and let $\mathcal{K}$ be a class of algebras of the same type. If $\mathbf{A}$ satisfies the general finite embedding property for $\mathcal{K}$ then $\mathbf{A} \in \operatorname{ISP}_{\mathrm{U}}(\mathcal{K})$.

Theorem 2. [3, Theorem 7] Let $\mathbf{A}=(A, \mathrm{~F})$ be an algebra such that $\mathrm{F}$ is finite and let $\mathcal{K}$ be a class of algebras of the same type. If $\mathbf{A} \in \mathrm{ISP}_{\mathrm{U}}(\mathcal{K})$ then $\mathbf{A}$ satisfies the general finite embedding property for $\mathcal{K}$.

\subsection{Farkas' lemma}

Let us recall the original formulation of Farkas' lemma [6, 7] on rationals:

Theorem 3 (Farkas' lemma). Given a matrix $A$ in $\mathbb{Q}^{m \times n}$ and $\boldsymbol{c}$ a column vector in $\mathbb{Q}^{m}$, then there exists a column vector $\boldsymbol{x} \in \mathbb{Q}^{n}, \boldsymbol{x} \geq \mathbf{0}_{n}$ and $A \cdot \boldsymbol{x}=\boldsymbol{c}$ if and only if, for all row vectors $\boldsymbol{y} \in \mathbb{Q}^{m}, \boldsymbol{y} \cdot A \geq \mathbf{0}_{m}$ implies $\boldsymbol{y} \cdot \boldsymbol{c} \geq 0$.

In what follows, we will use the following equivalent formulation:

Theorem 4 (Theorem of alternatives). Let $A$ be a matrix in $\mathbb{Q}^{m \times n}$ and $\boldsymbol{b}$ a column vector in $\mathbb{Q}^{n}$. The system $A \cdot \boldsymbol{x} \leq \boldsymbol{b}$ has no solution if and only if there exists a row vector $\boldsymbol{\lambda} \in \mathbb{Q}^{m}$ such that $\boldsymbol{\lambda} \geq \mathbf{0}_{m}, \boldsymbol{\lambda} \cdot A=\mathbf{0}_{n}$ and $\boldsymbol{\lambda} \cdot \boldsymbol{b}<0$.

\section{The Embedding Lemma}

In this section, we use the Farkas' lemma on rationals to prove that any finite partial subalgebra of a linearly ordered MV-algebra can be embedded into $\mathbb{Q} \cap[0,1]$ and hence into the finite MVchain $\mathbf{L}_{k} \subseteq[0,1]$ for a suitable $k \in \mathbb{N}$.

Lemma 1. Let $\mathbf{M}=(M ; \oplus, \neg, 0)$ be a linearly ordered $M V$-algebra, $X \subseteq M \backslash\{0\}$ be a finite subset. Then there is a rationally valued map $s: X \cup\{0,1\} \longrightarrow[0,1] \cap \mathbb{Q}$ such that

$$
\text { 1. } s(0)=0, s(1)=1 \text {, }
$$


2. if $x, y, x \oplus y \in X \cup\{0,1\}$ such that $x \leq \neg y$ and $x, y \in X \cup\{0,1\}$ then $s(x \oplus y)=s(x)+s(y)$.

3. if $x \in X$ then $s(x)>0$.

Lemma 2 (Embedding Lemma). Let us have a linearly ordered $M V$-algebra $\mathbf{M}=(M ; \oplus, \neg, 0)$ and let $X \subseteq M$ be a finite set. Then there exists an embedding $f: \mathbf{X} \hookrightarrow \mathbf{L}_{k}$, where $\mathbf{X}$ is a partial MV-algebra obtained by the restriction of $\mathbf{M}$ to the set $X$ and $\mathbf{L}_{k} \subseteq[0,1]$ is the linearly ordered finite $M V$-algebra on the set $\left\{0, \frac{1}{k}, \frac{2}{k}, \cdots, 1\right\}$.

\section{Extensions of Di Nola's Theorem}

In this section, we are going to show Di Nola's representation Theorem and its several variants not only via standard MV-algebra $[0,1]$ but also via its rational part $\mathbb{Q} \cap[0,1]$ and finite MVchains. To prove it, we use the Embedding Lemma obtained in the previous section. First, we establish the FEP for linearly ordered MV-algebras.

Theorem 5. 1. The class $\mathcal{L M V}$ of linearly ordered $M V$-algebras has the FEP.

2. The class $\mathcal{M V}$ of $M V$-algebras has the FEP.

Note that the part (1) of the preceding theorem for subdirectly irreducible MV-algebras can be easily deduced from the result that the class of subdirectly irreducible Wajsberg hoops has the FEP (see [1, Theorem 3.9]). The well-known part (2) then follows from [1, Lemma 3.7, Theorem 3.9]. We are now ready to establish a variant of Di Nola's representation Theorem for finite MV-chains (finite MV-algebras).

Theorem 6. 1. Any linearly ordered $M V$-algebra can be embedded into an ultraproduct of finite $M V$-chains.

2. Any $M V$-algebra can be embedded into a product of ultraproducts of finite $M V$-chains.

3. Any $M V$-algebra can be embedded into an ultraproduct of finite $M V$-algebras (which are embeddable into powers of finite $M V$-chains).

The next two theorems cover Di Nola's representation Theorem and its respective variants both for rationals and reals.

Theorem 7. 1. Any linearly ordered MV-algebra can be embedded into an ultrapower of $\mathbb{Q} \cap[0,1]$.

2. Any $M V$-algebra can be embedded into a product of ultrapowers of $\mathbb{Q} \cap[0,1]$.

3. Any $M V$-algebra can be embedded into an ultrapower of the countable power of $\mathbb{Q} \cap[0,1]$.

4. Any $M V$-algebra can be embedded into an ultraproduct of finite powers of $\mathbb{Q} \cap[0,1]$.

Theorem 8. 1. Any linearly ordered MV-algebra can be embedded into an ultrapower of $[0,1]$.

2. Any $M V$-algebra can be embedded into a product of ultrapowers of $[0,1]$.

3. Any MV-algebra can be embedded into an ultrapower of the countable power of $[0,1]$.

4. Any MV-algebra can be embedded into an ultraproduct of finite powers of $[0,1]$.

Proof. (1)-(4) It is a corollary of Theorem 6. 


\section{References}

[1] Blok, W., Ferreirim, I.: On the structure of hoops, Algebra Universalis 43, 233-257 (2000).

[2] Blok, W., van Alten, C. J.: On the finite embeddability property for residuated ordered groupoids, Trans. Amer. Math. Soc. 357, 4141-4157 (2005).

[3] Botur, M: A non-associative generalization of Hájeks BL-algebras, Fuzzy Sets and Systems 178, 24-37 (2011).

[4] Chang, C.C.: Algebraic analysis of many valued logics, Trans. Amer. Math. Soc. 88, 467-490 (1958).

[5] Di Nola, A.: Representation and reticulation by quotients of MV-algebras, Ricerche di Matematica XL, 291-297 (1991).

[6] Farkas, G.: Über die Theorie der einfachen Ungleichungen, Journal für die Reine und Angewandte Mathematik 124, 1-27 (1902).

[7] Schrijver, A.: Theory of linear and integer programming, Wiley-Interscience series in discrete mathematics and optimization, John Wiley \& sons (1998). 